\title{
Two steps towards the realization of fusion: New plasma configurations in the TCV tokamak and its ongoing upgrades
}

\author{
Yves Martina, Basil P. Duval, Alexander N. Karpushov, Benoit Labit, Holger Reimerdes \\ and TCV Team \\ Ecole Polytechnique Fédérale de Lausanne (EPFL), Centre de Recherches en Physique des Plasmas \\ (CRPP), Association EURATOM - Confédération Suisse, CRPP - EPFL, 1015 Lausanne, Switzerland
}

\begin{abstract}
To realise the potential of fusion as an abundant energy source, several challenges remain. The TCV tokamak, featuring high shaping capability and a flexible heating system, is strongly contributing to solving these challenges. A fundamental challenge remains in controlling heat exhaust from the plasma. ITER's currently foreseen operational regime implies heat flows to the plasma facing materials that are not compatible with a commercial fusion reactor. TCV has demonstrated alternative plasma divertor configurations, termed "snowflakes", that have the potential to strongly reduce the heat flux towards the vessel walls. Measurements of particle fluxes, together with IR camera imaging, show a clear reduction of the peak heat fluxes to the walls when the exhaust power is particularly large and a reduction of the heat fluxes most needed. Another challenge lies in the control of plasma instabilities and turbulence in reactor relevant operational regimes. To address this issue, TCV is presently complementing its electron heating system with an ion heating system: a $1 \mathrm{MW}$ neutral beam injector. With these ion and electron heating capabilities, TCV will be able to operate high temperature, reactor relevant, plasmas with of ion to electron temperature ratios.
\end{abstract}

\section{Introduction}

Fusion is the energy that powers the stars. In the sun, nuclei of Hydrogen fuse, eventually forming Helium and heavier atoms with additional kinetic energy. Each of these processes has an individual reaction rate that is a function of the local temperature and density. To reproduce fusion reaction on earth, one would opt for the Deuterium - Tritium (D-T) reaction as the necessary environmental parameters are most easily obtained. However, temperatures of the order of $100 \mathrm{M}^{\circ} \mathrm{C}$ are necessary to obtain a significant D-T fusion rate. Where gravitational forces keep matter in the sun confined, magnetic confinement is used on earth to maintain a high temperature D-T mixture that, at these temperatures, is in the plasma state. A tokamak is a toroidal device with a metallic vessel that surrounds the plasma, a toroidal solenoid that surrounds this vessel that produces the main toroidal field that confines the particles. A set of toroidal coils induce a current in the plasma that stabilises particle drifts due to the $1 / \mathrm{R}$ dependence of the toroidal field and an array of poloidal field coils shape the plasma confinement

\footnotetext{
${ }^{\mathrm{a} C}$ Corresponding author: yves . martin@epf $1 . \mathrm{ch}$
}

This is an Open Access article distributed under the terms of the Creative Commons Attribution License 4.0, which permits unrestricted use, distribution, and reproduction in any medium, provided the original work is properly cited. 


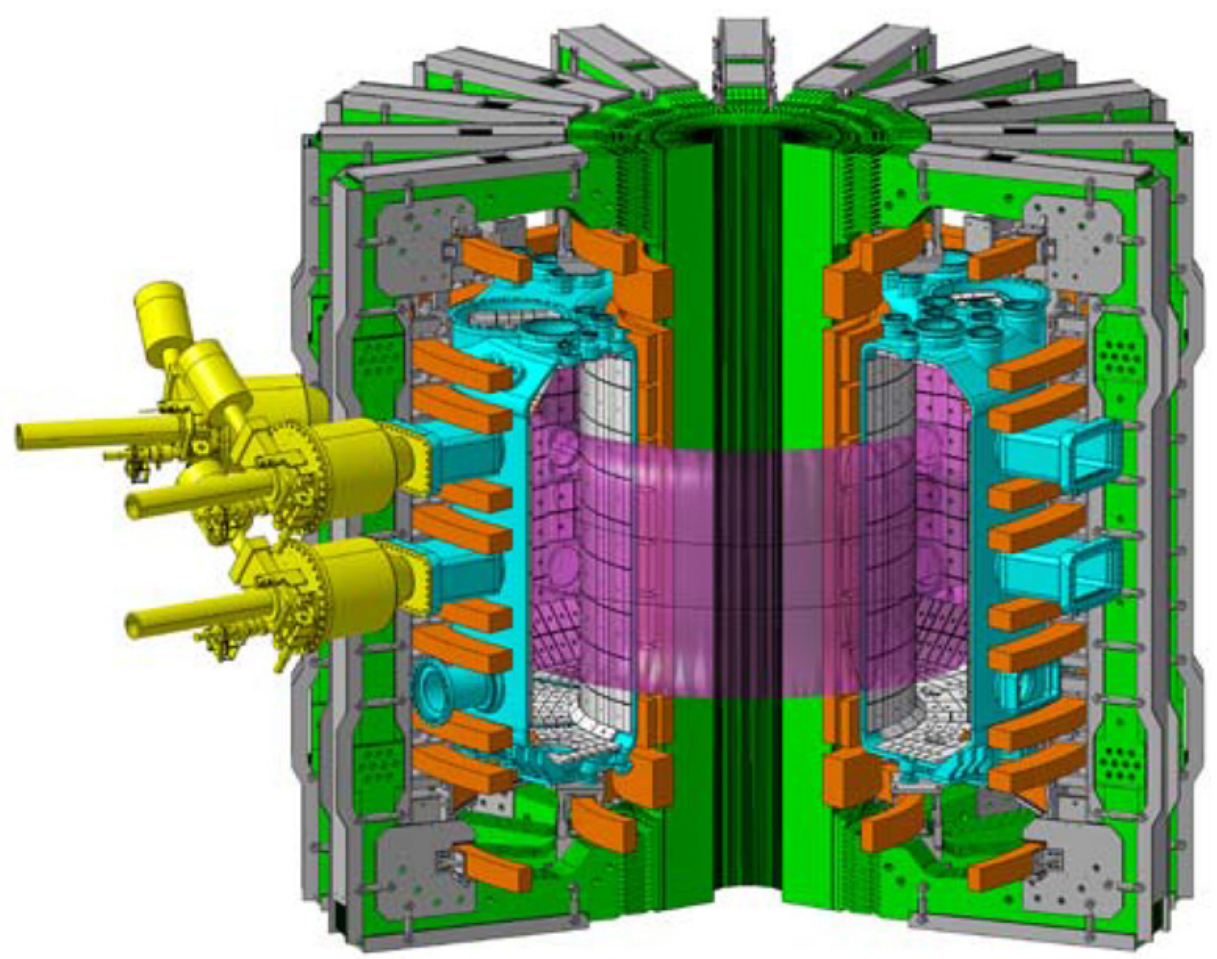

Figure 1. The TCV tokamak: vacuum vessel in cyan; toroidal field coils in green; poloidal field coils, for plasma current induction and plasma shaping, in orange; 3 out of 9 microwave launching systems in yellow. The plasma is represented in magenta.

region. Although the plasma current heats the plasma to temperatures of the order of $10 \mathrm{M}^{\circ} \mathrm{C}$, additional heating is required to achieve the temperatures required for fusion. To date, the concept of the tokamak is the most advanced amongst magnetic confinement schemes, so ITER, an experimental fusion reactor currently under construction, is based on the tokamak concept.

The Tokamak à Configuration Variable (TCV) at the Ecole Polytechnique Fédérale de Lausanne (EPFL) features 16, independently powered, poloidal field coils and a highly elongated vessel, as shown in Fig. 1, making TCV the tokamak with the highest plasma shaping capability in the world [1, 2]. It has a major radius of $0.9 \mathrm{~m}$, a minor radius of $0.25 \mathrm{~m}$. The vessel has an elongation (height over width ratio) of 3. The maximum achieved plasma current is $1 \mathrm{MA}$, while the toroidal field can reach $1.5 \mathrm{~T}$ at the centre of the machine.

TCV is equipped with a highly versatile Electron Cyclotron Resonance Heating (ECRH) system. Up to 9 gyrotrons (microwave sources, $500 \mathrm{~kW}$ each) deliver microwave beams to the plasma via launching systems using real-time steerable mirrors. The microwaves resonate with the electron cyclotron motion inducing strong and localised electron heating.

\section{A new configuration in TCV - the snowflake}

Two major classes of plasma configurations are operated in tokamaks. The first, and simplest, one, called 'limited configuration' includes all cases where the edge of the plasma directly lies against part of the vessel wall. The plasma periphery is then defined by the last closed flux surface, as shown in 
a)

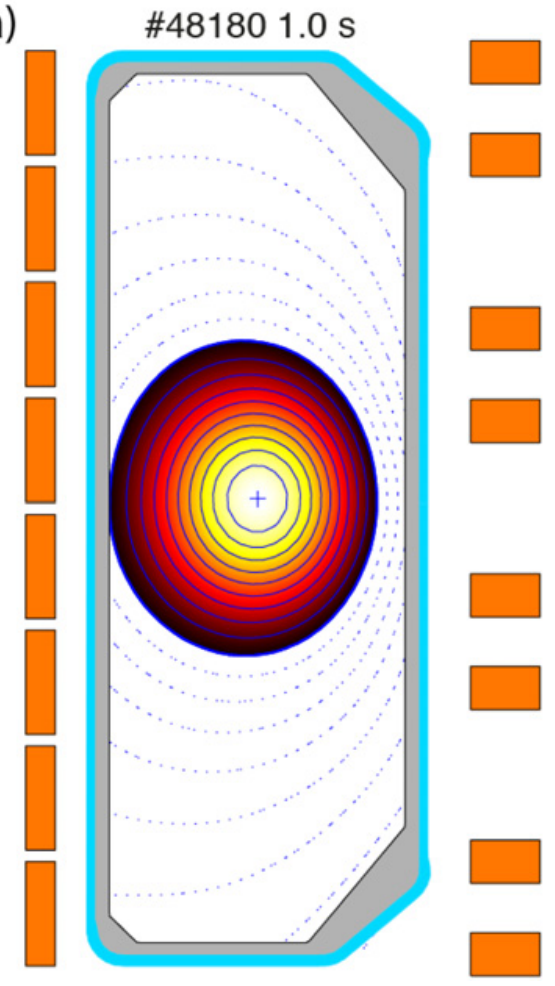

b)

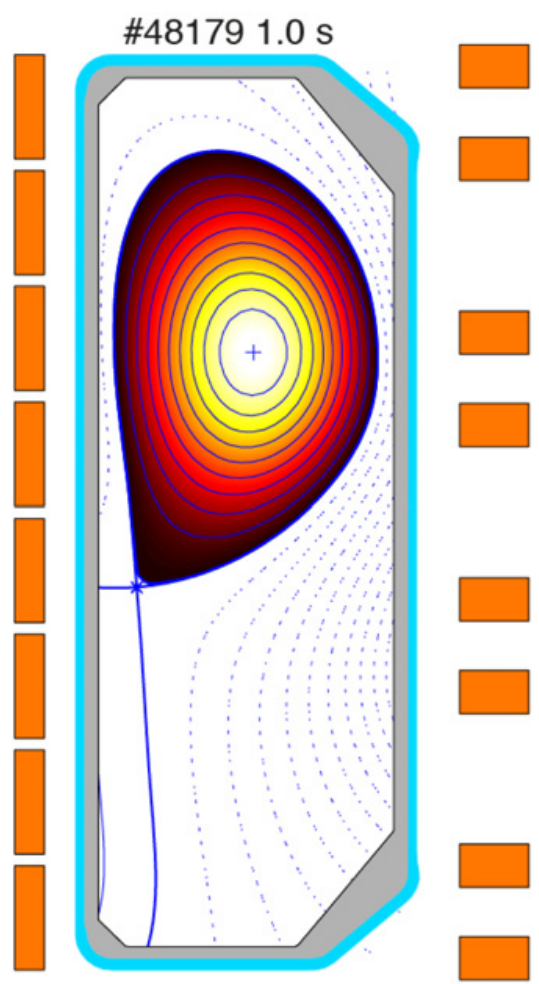

Figure 2. Plasma configurations obtained in TCV. The cross-section of the vessel is represented in cyan, while Carbon protection-tiles are in gray. A) Limited configuration. B) Diverted configuration. In B), the plasma, represented in false color, is clearly located at a distance from the walls. The solid blue line shows the separatrix legs that intersect the wall at two points.

Fig. 2a. The second category, termed "diverted configuration", aggregates plasma configurations that have an X-point, i.e. a location where the poloidal magnetic field vanishes defining a plasma separatrix that does not intersect the device wall, apart from the so-called "separatrix legs", as presented in Fig. 2b. This configuration entails several advantages. The increased distance between the plasma and the wall reduces the wall-plasma interaction and thus the impurities that may migrate from the wall to the plasma core. In addition, this configuration facilitates access to a high confinement regime, known as H-mode, where a significant reduction of the energy transport occurs at the plasma edge. However, these configurations also have a disadvantage: particles that escape from the plasma, via collisions for instance, flow toward the wall along the magnetic field lines, leading to high particle and energy fluxes where the separatrix legs intersect the wall.

In a fusion reactor, the heat flux associated with this particle flux would exceed present material limits. A solution to decrease the heat flux could consist in increasing the wall deposition area and this could be obtained by increasing the number of separatrix legs. This is the idea of the so-called snowflake divertor [3], which has been experimentally pioneered in TCV [4].

The snowflake configuration in TCV is obtained when not only the poloidal magnetic field locally vanishes, but when its first derivative also vanishes. In operational terms, it consists in superimposing two divertor X-points, as described above. Figure 3 shows, on the left, a schematic of an exact snowflake configuration, where both X-points perfectly superimpose. The name of the configuration comes from the similarity between this resulting hexapole configuration and the structure of a snowflake. 

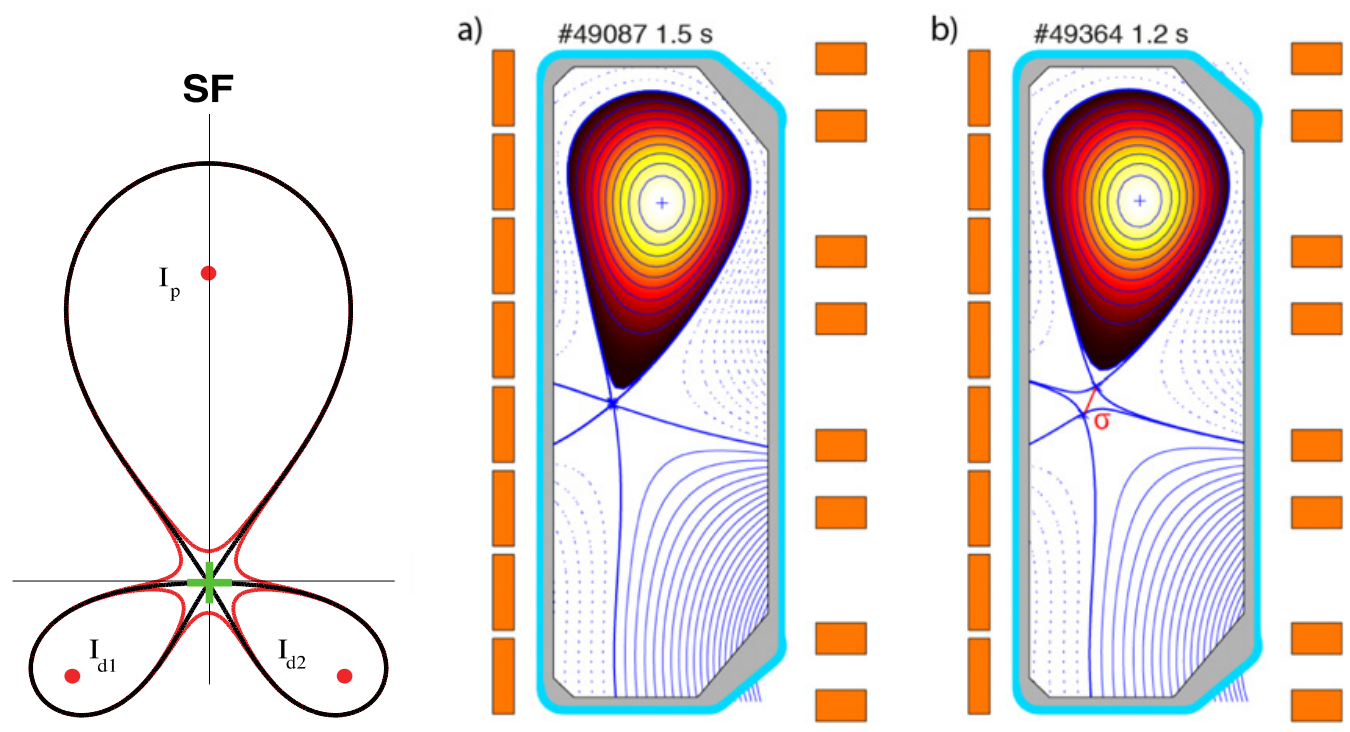

Figure 3. Left: schematic representation of an exact snowflake configuration with its hexapole structure that led to this denomination. Right: experimental results showing a) an exact snowflake configuration and b) a quasi snowflake with the distance between the two X-points indicated in red and labelled.

Since both lower lobes of the magnetic configuration are maintained by currents flowing in coils located outside the vessel, the separatrix legs now intercept the vessel wall at 4 locations, doubling the potential surface areas for heat flux deposition. In addition, Fig. 3, right, shows two experimental results: the first configuration shows an exact snowflake divertor with 4 separatrix legs while the second configuration shows a quasi snowflake, where the X-points are slightly separated.

The proximity of this configuration to an exact snowflake can be described by the parameter $\sigma$, that represents the distance between the X-points normalised to the plasma minor radius. Adjustment of the current flowing in the coils located at the bottom of both coil stacks, together with control of the plasma current and position are key to the control of the configuration.

TCV experiments are diagnosed by a series of measurement systems. Cameras sensitive to the visible light emitted by the plasma, are used to monitor the presence and location of the separatrix legs. An IR camera monitors the Carbon-tile temperature and is subsequently used to estimate the heat flux impinging onto these tiles. Sets of Langmuir probes, embedded in the vessel walls, diagnose the local plasma characteristics such density and temperature of the plasma reaching the wall.

All diagnostic systems see the 4-leg structure of the configuration. In low power discharges, the most of the power is exhausted along the two main divertor legs. However, while the measured power at the additional legs is only a small fraction of the total exhaust power, it is an order of magnitude larger than expectations based on transport assumptions typical for a conventional divertor [5]. This difference indicates an enhanced transport in the region around the two X-points where the poloidal field is particularly small. That should lead to significant heat flux reductions in a reactor-size tokamak [6].

A more advantageous power distribution to the additional divertor legs is observed in higher power discharges, which access the desired high confinement or H-mode regime [7]. H-modes are usually accompanied by periodic burst-like losses of energy, termed Edge Localised Modes or ELMs, that lead to transient, but particularly high heat fluxes at the wall. These ELMs can contain up to $10 \%$ of the total plasma energy and threaten the wall material. Nevertheless, the good energy confinement of 
$3^{\text {rd }}$ European Energy Conference

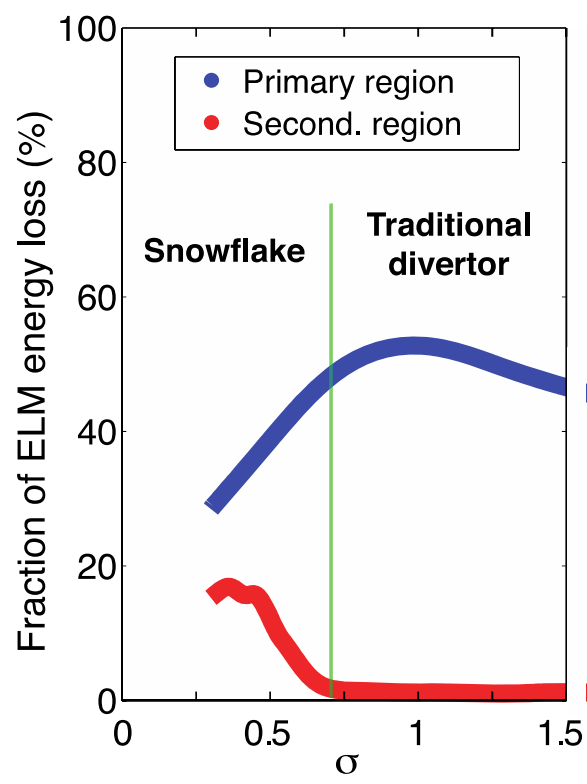

Figure 4. Fraction of the energy lost during ELMs towards one of the primary region separatrix leg and towards one of the secondary region, as a function of the relative distance between the two X-points.

the H-mode regime is still preferable for reactor operation and required to obtain high fusion energy production in ITER. Figure 4. summarises the distribution of power between a primary separatrix leg, in the upper region of the lobes, and a secondary separatrix leg in the region of the lower part of the lobes. About $50 \%$ of the total ELM energy loss reaches the primary region while negligible energy reaches the secondary one when the normalised distance between the two X-points is larger than 0.75 . For values below $0.75(\sim 17 \mathrm{~cm})$, the secondary $\mathrm{X}$-point becomes active and it is fully operational below approximately $0.3(\sim 7 \mathrm{~cm})$.

This figure clearly shows the benefit of the snowflake configuration, compared to a traditional diverted configuration, reducing the peak heat flux impinging onto the vessel wall during ELMs, i.e. when a reduction is most needed, by a factor of two.

\section{TCV upgrades}

To date, TCV has been equipped with a heating system, the Electon Cyclotron Resonance Heating (ECRH), which delivers power to the electrons, particularly at low density when the collision rate between electrons and ions is low. Although very interesting results were obtained, relevant to ITER, access to high pressure plasmas, with a range of temperature ratios (ion to electron temperature) is of prime importance to improve our knowledge of reactor-relevant plasmas. It is therefore desirable to equip TCV with an ion heating system [8].

The most effective way to heat ions in TCV is to inject high energy particles into the plasma and let them transfer their energy, mostly to the ions, collisionally. This method was developed in the early 1970s and is currently used on several tokamaks. These high energy particles must be neutral to penetrate the magnetic field surrounding the plasma. Fast neutral beams are generated via chargeexchange neutralisation of ion beams produced by extraction of ions from a suitable plasma source and acceleration to energies from several tens of kilovolts to $1 \mathrm{MeV}$. Beam neutral atoms are ionised inside the plasma through collisions with electrons and ions. 

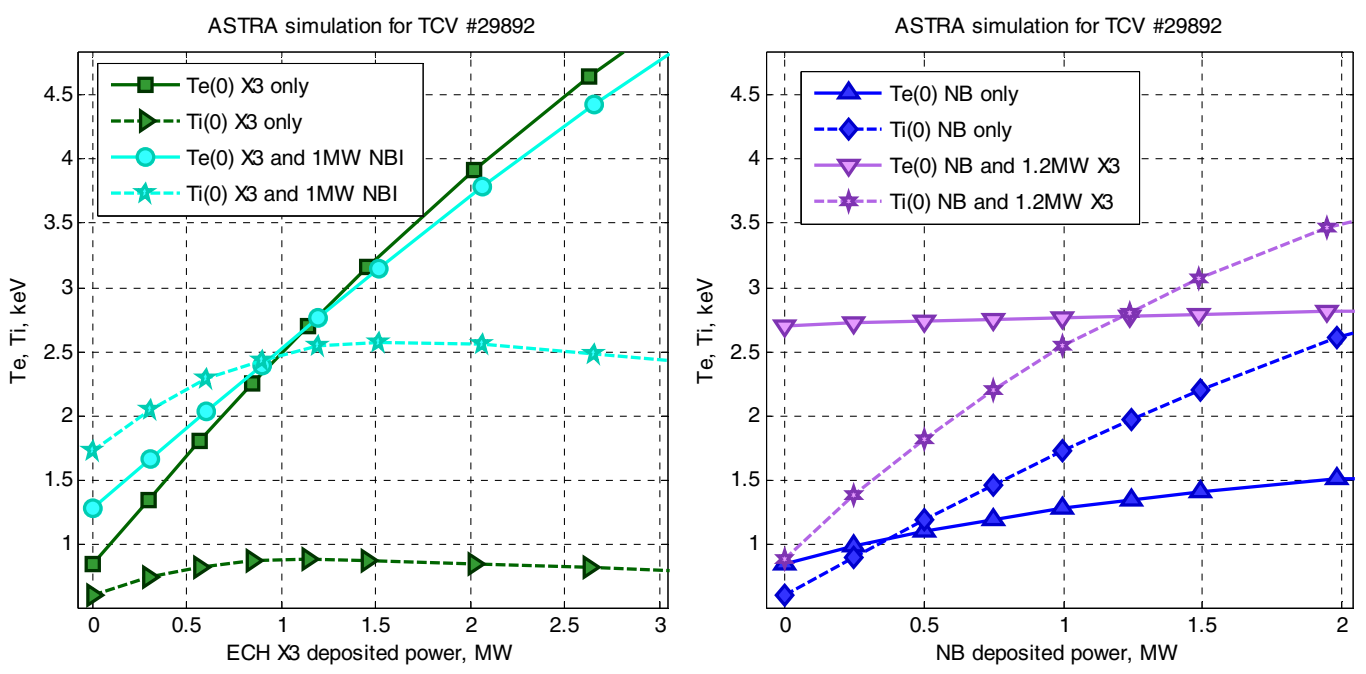

Figure 5. Variation of the electron and ion temperatures in the center of the plasma as a function of ECRH power (left) and NB power (right) from simulations based on an actual scenario.

Simulations have been performed to estimate the required characteristics of a Neutral Beam Injector (NBI) for TCV [9]. Ion energies of about $30 \mathrm{keV}$ are required to effectively heat dense TCV plasmas, without depositing excessive energy on the opposite wall. It must be noted that neutral beams are generally injected tangentially into tokamaks in order to increase the length of the beam-plasma interaction region to increase plasma absorption. Simulations, using parameters from actual TCV plasma cases (diverted plasma in H-mode with middle range density) show that the ion temperature should attain the electron temperature if about $1 \mathrm{MW}$ of NB power was absorbed in discharges already heated with 1.2 MW of X3 ECH, see Fig. 5. By varying the ECRH and NBI heating power, the ratio of ion to electron temperature is predicted to vary between 0.3 and 1.5 , as indicated in Fig. 5.

The power gain of a fusion reactor strongly depends on the plasma pressure since extremely high temperatures are required and the fusion reaction rate increases as the square of the plasma density. The simulations also indicated the normalised pressure that can be achieved in plasmas with both electron and ion heating. Figure 6 shows this normalised pressure, $\beta_{N}$, as a function of the ECRH power (X3 for $3^{r d}$ harmonic X-mode resonance) and NBI power. With ECRH heating only, one would obtain the pressure curve plotted in green. It is in agreement with the experimental value of discharge \#29475. This discharge did not, however, attain the pressure corresponding to the ITER scenario with a plasma current generated by induction. With $1 \mathrm{MW}$ of NBI injected into the plasma, $\beta_{N}$ should reach 2.0 , see Fig. 6. This would then correspond to the operational pressure regime of inductive discharges of ITER. Adding a further MW of NB or ECRH would permit the investigation of conditions corresponding to the Hybrid scenarios of ITER and test limitations of plasma pressure ( $\beta$ limit) in a machine where the plasma shape can be widely varied.

In a first step, it is planned to install a $1 \mathrm{MW}$ neutral beam injection system in TCV [10]. Preparatory work is presently on-going to allow the transformation of two radial ports on the current TCV vessel into tangential ports appropriate for beam injection. One of these ports will receive the beam and the other will be used for diagnostic purposes. In a second step, two additional gyrotrons of $1 \mathrm{MW}$ each, will be installed on TCV. In the future, it is considered to install a further $1 \mathrm{MW}$ neutral beam on the second tangential port. 

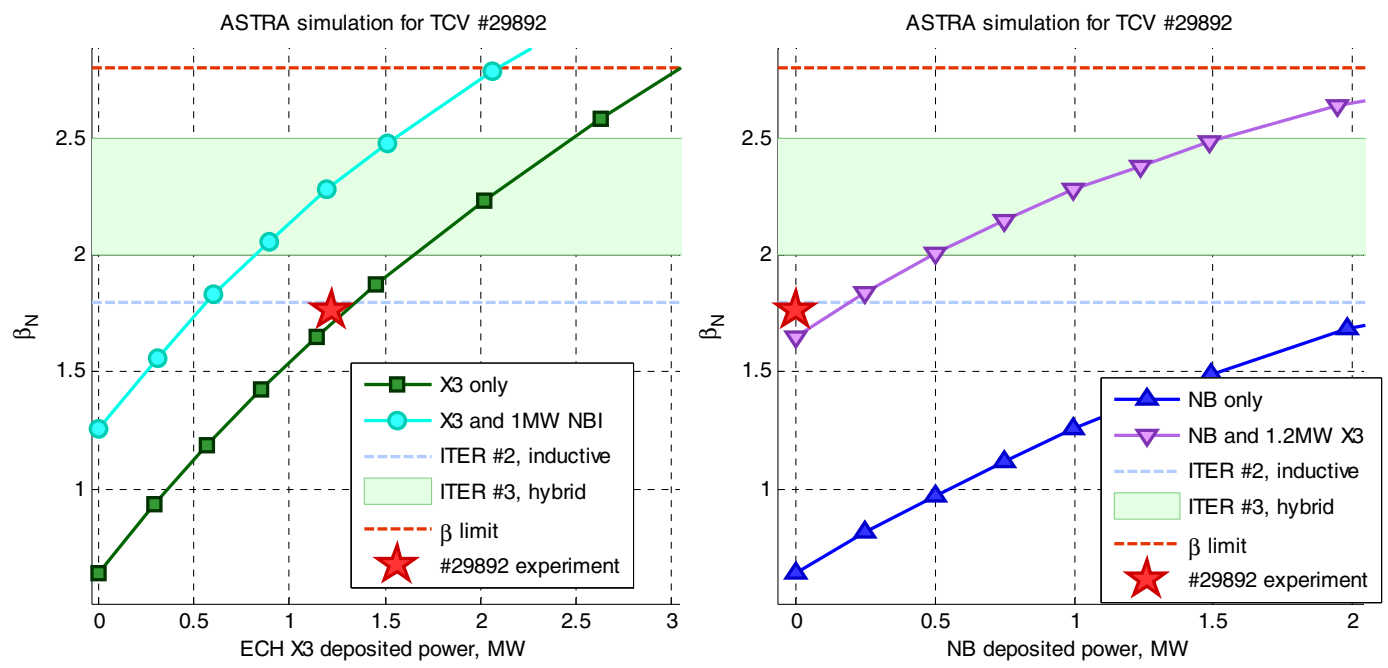

Figure 6. Normalised plasma pressure with different combination of plasma heating schemes as a function of EC $\mathrm{X} 3$ power (left) and NB power (right), for the same cases shown in Fig. 5.

Following these upgrades, TCV will be fully equipped to address several pending issues related to burning plasmas, will gain relevance to much larger and higher cost plasmas with a significant fusion power yield.

\section{Conclusions}

TCV is a unique worldwide device, that can address important issues related to burning plasmas, thanks to its high shaping capability and highly versatile ECRH heating system. It has already demonstrated a possible reduction of the heat flux deposited onto the tokamak wall by shaping the plasma into the snowflake configuration. The addition of $1 \mathrm{MW}$ of neutral beam will widen the TCV operational domain to fully access the reactor relevant regimes.

This work was supported in part by the Swiss National Science Foundation.

\section{References}

[1] F. Hofmann et al., Plasma Phys. Control. Fusion 36, B277 (1994)

[2] S. Coda et al., Nucl. Fusion 53, 104011 (2013)

[3] D. D. Ryutov, Phys. Plasmas 14, 064502 (2007)

[4] F. Piras et al., Plasma Phys. Control. Fusion 51, 055009 (2009)

[5] T. Lunt et al., submitted to Plasma Phys. Control. Fusion

[6] H. Reimerdes et al., Plasma Phys. Control. Fusion 55, 124027 (2013)

[7] W. Vijvers et al., submitted to Nucl. Fusion

[8] A. Fasoli et al., 40 ${ }^{\text {th }}$ EPS Conf. on Plasma Physics, P2.104 (2013)

[9] A. N. Karpushov et al., Fusion Engineering \& Design 86, 868 (2009)

[10] A. N. Karpushov et al., 36 ${ }^{\text {th }}$ EPS Conf. on Plasma Physics, P2.140 (2009) 